Man and Nature

MAN AND NATURE

L'homme et la nature

L'HOMME ET LA NATURE

\title{
Les Fantasmes de l'argent dans l'Histoire du chevalier des Grieux et de Manon Lescaut
}

\section{Raymond Joly}

Volume 1, 1982

URI : https://id.erudit.org/iderudit/1011786ar

DOI : https://doi.org/10.7202/1011786ar

Aller au sommaire du numéro

Éditeur(s)

Canadian Society for Eighteenth-Century Studies / Société canadienne d'étude du dix-huitième siècle

ISSN

0824-3298 (imprimé)

1927-8810 (numérique)

Découvrir la revue

Citer cet article

Joly, R. (1982). Les Fantasmes de l'argent dans l'Histoire du chevalier des Grieux et de Manon Lescaut . Man and Nature / L'homme et la nature, 1, 1-13.

https://doi.org/10.7202/1011786ar

Copyright (C Canadian Society for Eighteenth-Century Studies / Sociéte canadienne d'étude du dix-huitième siècle, 1982
Ce document est protégé par la loi sur le droit d'auteur. L'utilisation des services d'Érudit (y compris la reproduction) est assujettie à sa politique d'utilisation que vous pouvez consulter en ligne.

https://apropos.erudit.org/fr/usagers/politique-dutilisation/ 


\section{Les Fantasmes de l'argent dans $l$ 'Histoire du chevalier des Grieux et de Manon Lescaut}

"Il n'y a proprement de Français en France que le petit nombre de ceux qui sont à la tête des autres, et qui sont distingués de ce qu'on appelle peuple." Ainsi s'exprime Cleveland, le Philosophe anglais, au livre II de ses mémoires. C'est la même conclusion qu'impose l'Histoire du chevalier des Grieux et de Manon Lescaut.

Le marquis de Renoncour, l'Homme de qualité à qui nous sommes redevables de ce récit (écrit, pour ainsi dire, sous la dictée du héros ${ }^{2}$ ), nous renseigne dès l'entrée en matière sur les circonstances de sa première rencontre avec des Grieux:

Je revenais un jour de Rouen, où [ma fille] m'avait prié d'aller solliciter une affaire au Parlement de Normandie pour la succession de quelques terres auxquelles je lui avais laissé des prétentions du côté de mon grandpère maternel [donc, notons-le en passant, de sa mère à lui] (p. 10).

Il appartient à la classe dominante, dominante par le privilège de la noblesse, mais aussi par la possession héréditaire de ce qui est encore, dans la France de cette époque, la principale source de la richesse, à savoir le sol cultivable. Tout indique que la famille des Grieux, l'"une des meilleures maisons de P." (p. 17), est de même rang.

Le reste du monde, aux yeux des représentants de cette classe, consiste en animaux plus ou moins grossiers. Les financiers sont de dégoûtants personnages, qui ne sauraient faire partie du cercle des connaissances de des Grieux père, et à qui il prend bien soin de refuser la particule (pp. 33-34). Son fils, quant à lui, trouve normal de les duper et s'indigne qu'ils osent le lui reprocher; surpris en flagrant délit de proxénétisme et d'escroquerie, son sang noble, juge-t-il, l'autorise encore à réclamer le châtiment de ses victimes (pp. 153-156). La naissance de des Grieux, comparée à celle de ses ennemis, l'innocente pratiquement aux yeux du supérieur de Saint-Lazare et du lieutenant de police aussi (pp. 83sqq., 113, 160-161). La bourgeoisie n'est représentée que par l'estimable famille Lescaut et, sans doute, par Tiberge, le personnage le plus systématiquement bafoué du livre. Quant au peuple, il se ramène en somme à la populace stupide qui gêne des Grieux et l'Homme de qualité dans leurs mouvements (pp. 10-11), aux lâches coquins que sont les 
archers (pp. 13, 15, 174), à ces bêtes de cirque, les prostituées (p. 178), à l'imbécile portier de Saint-Lazare qui voulait empêcher l'évasion de des Grieux, et qui n'a pas à se surprendre si on lui brûle la cervelle (p. 97), et aux domestiques. Ceux-ci se divisent en deux catégories, selon qu'ils appartiennent au père des Grieux (auquel cas ils sont bons, dévoués et incorruptibles [pp. 21, 35-36]) ou à d'autres maîtres; ce sont alors des espèces d'enfants, susceptibles de réactions affectives primaires, qu'on achète facilement mais qui trahissent pour des raisons aussi futiles que le risque d'être pendus, et toujours suspects de vouloir voler leurs maîtres (pp. 13, 66-67, 104, 120, 154). Enfin, l'histoire de la vocation de des Grieux (pp. 40-42) et ses rapports avec les membres du clergé, dont Tiberge, montrent avec la plus grande clarté que le rôle de l'institution ecclésiastique est essentiellement de servir les intérêts de la noblesse, notamment en ouvrant aux cadets de famille une possibilité d'ascension sociale.

En bref, il n'y a d'hommes dignes de ce nom que ceux qui appartiennent à l'aristocratie. Ce point de vue, confirmé par tout le roman, s'exprime dès les premières pages. J'en citerai comme exemple le beau jugement du marquis de Renoncour, qui commente en ces termes les témoignages de gratitude du héros, à qui il vient de procurer, au prix de quelques louis d'or, le droit de rester aux côtés de Manon jusqu'à l'embarquement pour le Nouvel Orléans: "La bonne grâce et la vive reconnaissance avec laquelle ce jeune inconnu me remercia, achevèrent de me persuader qu'il était né quelque chose, et qu'il méritait ma libéralité" (p. 15; voir aussi p. 81). Les bonnes manières et les qualités du coeur s'expliquent par la naissance, et la naissance donne droit aux bienfaits - lesquels, sommes-nous autorisés à conclure, seraient mal employés s'ils tombaient sur des êtres qui n'ont ni naissance, ni, par conséquent, qualités du coeur et de l'esprit.

Le malheur, c'est que l'Histoire de des Grieux n'est pas un roman de Chrétien de Troyes, de La Calprenède ou de Mme de la Fayette. L'idéologie féodale est une bien médiocre boussole pour s'orienter dans la société de la Régence, surtout quant on ne fait pas partie, quoi qu'on veuille se faire accroire, de la classe dominante. Des Grieux oscille entre deux mondes, celui où l'on vaut ce qu'on a et celui où l'on vaut ce qu'on est/naît, sans avoir accès ni à l'un ni à l'autre.

On a souvent commenté la stupeur fascinée du héros devant ces abîmes incompréhensibles que sont l'amour, les femmes, Manon. Je ne sais pas si le mystère est vraiment si impénétrable que cela; ce qui est sûr, c'est que l'univers de des Grieux comporte une autre donnée tout aussi irrationnelle, une autre force devant laquelle il reste absolument désarmé, et qui est l'argent. Il essaie désespérément et maladroitement de calculer, de compter; il en est obsédé au point de faire des équations pour vérifier qu'une moitié de son sang plus une autre partie font bien son sang tout entier (pp. 111-112); le monde qui l'entoure a l'air de fonctionner sur le principe que tout s'échange, que tout s'achète. Mais ce qui lui échappe complètement, c'est l'origine de la richesse. Défavorisé, pour ce qui est des héritages, par sa condition de cadet, ignorant qu'il existe une réalité appelée travail (dans tout le roman, d'ailleurs, on ne trouve pas un seul représentant des classes productrices), des Grieux observe le va-et-vient de l'argent, ses apparitions miraculeuses et ses 
disparitions par enchantement, comme autant de mouvements sans cause. Logiquement, son seul projet suivi en la matière est d'entretenir Manon au moyen du jeu.

Il faut cependant parler un peu plus en détail de l'héritage, que nous venons d'évoquer. Le budget fort irréaliste de l'ex-abbé de Saint-Sulpice s'établit sur une base de dix ans, au cours desquels, dit-il à Manon, il est impossible qu' "il n'arrive point de changement dans ma famille; mon père est âgé, il peut mourir. Je me trouverai du bien, et nous serons alors au-dessus de toutes nos autres craintes" (p. 50). Trois pages plus loin, il est de nouveau question de ces "changements que j'espérais," allusion bien pudique au désir de tuer le père. Cette mort, au surplus, ne réglerait pas le problème, puisque le vieillard, qui était prêt à vouer son fils au célibat en le destinant à l'ordre de Malte ou à l'état ecclésiastique (p. 18), se survit en un fils aîné, autre luimême qui avait fort bien fait ses preuves dans l'épisode de la première trahison de Manon, quand il s'était agi d'enlever le frère cadet à Paris et de l'emmener réfléchir quelques mois à la maison, sous bonne garde. Pas grandchose, donc, à espérer de ce côté-là; aussi bien, quand des Grieux reviendra du Nouvel Orléans, moralement régénéré et définitivement débarrassé de sa catin, il s'en faudra que la maison paternelle s'ouvre toute grande au fils repentant: l'aîné, devenu entre-temps chef de la famille, donne rendez-vous à son frère hors de chez lui, dans la maison d'un parent (p. 204). Reste donc l'autre partie de l'héritage, la seule sur laquelle il faille vraiment compter: "étant dans ma vingtième année, j'entrais en droit d'exiger ma part du bien de ma mère" (p. 117).

Avouons qu'il y a une logique derrière ce rejet que les instances paternelles infligent au Chevalier. En admettant qu'il en eût les moyens matériels, des Grieux ne pourrait prétendre sérieusement à l'intégration dans la classe à laquelle il appartient par sa naissance, ni à la succession. Le roman et son héros ont beau se cramponner à l'idéologie aristocratique, ils n'en présentent en fait qu'une image dérisoire: parodie de la noblesse dans les rituels de l'ordre chevaleresque des escrocs qui détroussent les joueurs à l'hôtel de Transylvanie (pp. 62-64), cynisme de des Grieux lorsqu'il ramène la société à deux classes, les riches stupides et les pauvres astucieux, et nous montre que la vocation providentielle des seconds consiste à sucer l'argent des premiers, en se faisant leurs maquereaux ou leurs précepteurs de vertu - indifféremment, car l'un vaut l'autre (pp. 53-54). La déchéance du Chevalier se marque surtout, et non plus épisodiquement, par un détournement radical de l'idée même de noblesse.

Il serait simpliste de dire que la classe dominante, dans une société donnée, ne fait rien d'autre que de consommer le produit du travail de celles qu'elle exploite, si significative et si fondamentale que soit au demeurant cette consommation publiquement revendiquée. La classe dominante joue aussi d'autres rôles; même si l'analyse de son idéologie révèle qu'elle les survalorise, qu'elle cherche systématiquement à dénier que quiconque puisse l'y remplacer, qu'elle en invente pour se rendre indispensable; bref, même si la vocation qu'elle s'attribue découle moins de la nature des choses que de la façon dont elle a intérêt à voir les choses, il n'en reste pas moins qu'elle fonde sa légitimation - sinon sa légitimité - sur une responsabilité sociale. La 
classe dominante ne peut réclamer le pouvoir sans se montrer prête à l'exercer effectivement.

Dans le cas de la noblesse d'Ancien Régime, nous savons très bien ce que cela veut dire. Malgré les transformations qu'a subies la féodalité du fait de la montée du pouvoir royal, le noble est toujours un guerrier; il est toujours seigneur, rentier et protecteur de ses vassaux; la participation à l'administration de la justice et à la gestion des affaires publiques continue à faire partie de ses prérogatives héréditaires, même si Versailles les menace. Enfin, sa responsabilité idéologique demeure impérieuse: il est le gardien des valeurs de sa classe, donc des valeurs dominantes de toute la société.

Est-il besoin de dire qu'une conception de la noblesse qui consiste à ruer dans les brancards parce qu'on n'a pas assez d'argent pour risquer plus de deux pistoles au jeu et pour emmener sa maîtresse à l'Opéra deux fois par semaine (pp. 49-50) semble un peu étriquée? Des Grieux se place en marge de l'aristocratie en n'en retenant comme signe distinctif que l'une des formes de la consommation, le divertissement, et surtout en refusant de reconnaître que la vie de l'homme de qualité, par définition, n'est pas que privée (erreur où ne tomberait pas un libertin).

Nous connaissons aussi fort bien le mode sur lequel la condition aristocratique pouvait se vivre subjectivement, et je ne sache pas que ce que Montesquieu écrivit sur l'honneur ait rien perdu de son intérêt. Si la noblesse est la classe qui se déclare prête à risquer sa vie pour le roi, et qui revendique en retour la participation au pouvoir, la richesse, les privilèges et le droit de définir les valeurs dominantes, l'homme de qualité est celui qui se déclare prêt à risquer sa vie pour son honneur et qui réclame en retour d'être reconnu par autrui.

On sait enfin - et c'est par là que je terminerai ce rappel de notions familières - quel lien s'établit dans l'idéologie aristocratique entre l'honneur et l'amour. L'accomplissement du héros, c'est-à-dire de l'idéal du noble, s'atteint lorsque coïncident la reconnaissance de soi comme être social et comme être sexué, reconnaissance qui n'est valide que si le partenaire est digne qu'elle soit réciproque. $M$. des Grieux père illustre admirablement ce point de vue. Révéler à son fils que c'est Manon elle-même qui s'est vendue à M.B. . . et qui s'est arrangée pour se débarrasser de son amant impécunieux, est à ses yeux un moyen infaillible pour détacher immédiatement l'aveugle, à qui il connaît des "principes d'honneur," d'un objet méprisable; il n'en revient pas lorsque cette manoeuvre échoue, et il reste incapable de croire à une passion aussi invraisemblable (pp. 36-37). De même, à l'autre bout du roman, s'il écoute d'une oreille attendrissable des allusions générales aux ardeurs amoureuses qu'il a pu éprouver dans sa jeunesse (p. 162), il se redresse sous l'outrage lorsque son fils ose comparer sa passion pour Manon à l'amour que lui, le père, avait voué à sa femme. C'est là-dessus que se rompt définitivement le contact entre le père et le fils (p. 172).

C'est dans ce problème de la double reconnaissance - sociale et sexuelle que je situerais le conflit fondamental du roman. La donnée de base en est que des Grieux doit renoncer à l'une ou à l'autre. Certes, son père, qui n'est pas un monstre, envisage un instant de le marier, croyant qu'il ne peut pas se passer de femme (p. 37); mais cette offre, aussitôt repoussée par le héros, reste 
sans poids, puisque la seule épouse qu'il puisse accepter est Manon (pp. 22, 26 , etc.), qui est exclue, à cause de sa condition et de ses moeurs. D'autre part, des Grieux, chevalier de Malte ou ecclésiastique, aurait pu, pour employer ses propres termes, "n'être pas plus scrupuleux qu'un grand nombre d'évêques et d'autres prêtres, qui savent accorder fort bien une maîtresse avec un bénéfice" (p. 65). Mais les conseils sarcastiques qui sont bons pour Tiberge ne le sont pas pour lui; ce qui serait vraisemblable dans les moeurs de l'époque n'est pas vrai pour le des Grieux réel, c'est-à-dire l'être textuel qui habite le roman de Prévost. Ici, il n'a d'autre alternative que de renoncer à la reconnaissance sexuelle en entrant dans les ordres (militaires ou non), ou à la reconnaissance sociale, en tournant le dos aux seules carrières qui s'ouvrent à un cadet.

En fin de compte, nous le savons, il n'obtiendra ni l'une ni l'autre. Le roman détaille toutes les étapes de la déchéance du Chevalier, et il la figure, comme allégoriquement, en rapprochant deux images: celle du pauvre hère, obligé de vendre sa monture pour suivre une déportée et rentrant d'Amérique, "cavalier désarçonné," ${ }^{3}$ ses guenilles sous le bras, et celle du gamin fringant qui caracole à côté de la chaise où la petite Manon vole vers Paris, comme pour une partie d'amour buissonnier (pp. 14, 16, 183; 24).

Le tableau amoureux ne me semble pas moins sombre, car sans cesse Manon renvoie son amant à sa nullité. Elle l'aime, dit-il et dit-elle; nous n'avons aucun droit de les contredire. Mais elle ne peut supporter l'idée de vivre petitement, de renoncer au plaisir et aux passe-temps.

Quoiqu'elle m'aimât tendrement, et que je fusse le seul, comme elle en convenait volontiers, qui pût lui faire goûter parfaitement les douceurs de l'amour, j'étais presque certain que sa tendresse ne tiendrait point contre de certaines craintes [la "crainte de la misère", c'est-à-dire surtout l'idée de renoncer à sa voiture, comme cela sera expliqué un peu plus loin, p. 70]. Elle m'aurait préféré à toute la terre avec une fortune médiocre; mais je ne doutais nullement qu'elle ne m'abandonnât pour quelque nouveau B. . . lorsqu'il ne me resterait que de la constance et de la fidélité à lui offrir (pp. 61-62).

Même la frêle assurance qui vient d'être citée s'évanouit d'ailleurs dans la suite: les vivres allaient manquer pour vrai lorsque Manon accueillit les avances de M. B. . ., mais c'est bel et bien l'éblouissement du luxe qui la fait se donner à G. . . M. . . fils, transformant en trahison de l'amour pour l'argent ce qui, au départ, ne devait être qu'une plaisante escroquerie.

Dans son rapport avec Manon, des Grieux apprend donc à se définir comme l'homme à qui il manque quelque chose, puisque ce qu'il est, et qu'elle aime, est impuissant à la satisfaire. Et, par une terrible ironie, elle ne cesse de lui infliger le renouvellement de sa castration. L'argent dont la possession pourrait lui assurer le bonheur dont il rêve, c'est à des figures paternelles qu'elle le demande: à M. B. . ., qui aura tôt fait de se lier avec le père des Grieux pour volatiliser son insignifiant rival - et notre héros, en pénitence au château paternel, retournera à ses livres d'écolier (p. 38); au vieux G. . . M. . . ensuite, ce qui donne lieu à un véritable festival de l'infantilisation: pour introduire son greluchon dans la maison du protecteur, 
Manon le fait passer pour son petit frère, fraîchement arrivé de province pour se préparer à entrer au séminaire; le cher enfant n'a pas d'autre plaisir que de "faire de petites chapelles" (pp. 76-77). C'est des Grieux lui-même, le des Grieux de Saint-Sulpice, qui se caricature ici sous les traits de l'eunuque demeuré, jeu qui durera jusqu'à ce que la figure paternelle terrible se démasque, chasse du lit l'impuissant et l'envoie dans une autre maison religieuse, la prison de Saint-Lazare.

La troisième trahison a ceci de particulier que des Grieux s'en fait lui-même le complice, qu'il aide à la préparer, tout en en prévoyant fort bien les suites. Autre particularité: ce n'est plus un vieillard qu'il s'agit d'exploiter, mais un jeune homme, l'ami fort estimable de cet incomparable ami qu'est $M$. de T. . . Le texte insiste sur les similitudes et les affinités qui font de celui-ci un double du héros - mais un double idéal, puisqu'à la jeunesse, à la noblesse, à la sensibilité et à la générosité de des Grieux il ajoute la pleine possession de tous les avantages de l'aristocratie: il est riche, et la première chose que nous apprenions de lui, c'est qu'il est "en âge d'être marié" (p. 99). Il doit cette situation enviable au fait d'être le fils de son père, personnage considérable et administrateur de l'Hôpital, mais ce père, pour comble de bonheur, est absent du roman. C'est le jeune M. de T. . ., donc, qui introduit dans le ménage clandestin de nos deux amants un autre lui-même, G. . . M. . . fils, en le présentant comme l'un de ses meilleurs amis, comme un homme d'honneur et un homme très aimable (p. 125). A voir ces trois garçons ensemble, festoyant à Chaillot avec la belle Manon, on dirait trois frères. Mais G. . . M. . ., lui, a un père pour vrai, que nous connaissons, comme nous connaissons celui de des Grieux.

L'opération érotico-financière qui se trame à partir de là n'a pas le même sens que les précédentes. La première fois, Manon avait agi seule; la seconde, elle avait eu recours à ce frère-janus, Lescaut, qui est le sien par le sang, mais qui sait fort bien se faire passer pour celui du Chevalier quand il s'agit d'introduire un pistolet dans sa cellule à Saint-Lazare; ici, des Grieux est de la partie. D'autre part, le plan ne comporte pas, cette fois-ci, de prostitution réelle: Manon est censée s'évader de la Comédie avant que le jeune G. . . M. . . ait commencé à profiter de son acquisition. Enfin, le but poursuivi est double: renflouer les finances du couple, venger les avanies infligées par G. . . M. . . père.

On peut donc interpréter l'épisode comme suit: des Grieux est toujours à la recherche de ce qui pourrait pallier sa castration, et il croit avoir trouvé l'astuce. Non seulement l'opération pourra se réaliser sans attaquer de front l'instance paternelle, mais revanche sera prise sur les humiliations antérieures. Ce qui manquait ne manquera plus, et ce ne sera pas parce que Manon l'aura soutiré au père en sacrifiant des Grieux; c'est bien des Grieux lui-même, sous les traits de son double, qui le lui aura donné; des Grieux aura coïncidé, dans son double, avec la seule image qu'il puisse tolérer de soimême: le jeune homme de bonne famille, libre, opulent et aimé.

La catastrophe est épouvantable. Voyons-en d'abord le dernier volet. Devant l'outrage infligé au père, les nuances de rang disparaissent. Des Grieux discute sur la noblesse et la pureté de son sang relativement à celui de ses victimes, regarde de haut M. de G. . M. .., ce "vieux monstre 
d'incontinence" (p. 153). Des Grieux père, lui, trouve normal de rendre visite à ce prétendu coquin, de faire alliance avec lui, d'humilier son fils devant cet homme respectable et offensé, qui s'exprime d'ailleurs, dans la circonstance, de la façon la plus mesurée, la plus digne et la plus patriarcale (p. 165). La victoire de l'autorité est complète; des Grieux retrouve un père-geôlier ( $\mathrm{pp}$. 165, 170, 172); Manon est expédiée, littéralement, au bout du monde.

Mais ce qui s'était déroulé auparavant est peut-être pire encore. Manon, on s'en souvient, n'eut pas plus tôt compris l'étendue des bienfaits de son nouveau protecteur qu'elle envoya promener son inutile amant. Au lieu d'exécuter la manoeuvre convenue pour le rejoindre, elle dépêcha à des Grieux l'ancienne maîtresse du jeune G. . . M. . ., pour le consoler en lui faisant passer un bon moment. La scène qui suit est étonnante (p. 133 sqq.). La rencontre a lieu dans un fiacre, à la nuit tombée, et la remplaçante est porteuse d'un billet de Manon. Que l'amant inquiet brûle de le lire, qu'il entre, pour ce faire, dans un cabaret, cela paraît normal, si l'on songe à ce qu'était alors l'éclairage nocturne des rues de Paris. On comprend moins bien pourquoi il s'installe avec la messagère dans un cabinet particulier. La pauvre enfant était payée pour une tâche précise, mais son client ne songe qu'à se livrer à des manifestations de douleur et de rage impuissante, décrites dans des termes qu'un esprit moyennement mal tourné rapportera sans peine à une autre sorte d'efforts inutiles.

Dans ce contexte, les mots que le héros adresse à sa consolatrice abasourdie ont un double sens parfaitement clair:

On t'a trompée, lui dis-je; ma pauvre fille, on t'a trompée. Tu es une femme, il te faut un homme; mais il t'en faut un qui soit riche et heureux, et ce n'est pas ici que tu le peux trouver. Retourne, retourne à M. de G. . . M. . . Il a tout ce qu'il faut pour être aimé des belles; il a des hôtels meublés et des équipages à donner. Pour moi, qui n'ai que de l'amour et de la constance à offrir, les femmes méprisent ma misère et font leur jouet de ma simplicité (p. 137).

Il peut retourner à ses petites chapelles; sa "simplicité" et sa pauvreté font qu'il n'est pas un homme. La fin du roman nous le montre d'ailleurs dans les bras du seul être qui l'ait aimé fidèlement, de son vrai double, de son vrai alter ego: $\mathrm{M}$. l'abbé Tiberge, chaste poire.

Nous avons donc lu le récit autobiographique d'une homme qui se juge castré, c'est-à-dire dépossédé de son propre corps. Un trouble pareil dans la fantasmation serait-il isolé? Faut-il s'attendre que l'image de la femme, elle, soit normale? C'est ce que nous examinerons maintenant.

Chaussons à nouveau nos besicles historico-sociologiques de tout à l'heure et demandons-nous ce qu'il en est de Manon comme corps social, capable d'entrer dans le cycle des échanges matrimoniaux. L'attitude de des Grieux à cet égard peut sembler fluctuante, mais je retiendrai surtout deux de ses déclarations, situées aux deux bouts du livre; elles me paraissent mériter une créance particulière parce que ce qui y paraît jaillit avec la force du lapsus, et se trouve en profond accord avec le mouvement général du récit.

La première exige, pour être bien comprise, d'être replacée dans son 
contexte. Nous sommes dans la cour de l'hôtellerie d'Amiens, au moment de la première rencontre. Je demandai à Manon, dit des Grieux,

ce qui l'amenait à Amiens et si elle y avait quelques personnes de connaissance. Elle me répondit ingénument qu'elle y était envoyée par ses parents pour être religieuse. L'amour me rendait déjà si éclairé, depuis un moment qu'il était dans mon coeur, que je regardai ce dessein comme un coup mortel pour mes désirs. Je lui parlai d'une manière qui lui fit comprendre mes sentiments, car elle était bien plus expérimentée que moi. C'était malgré elle qu'on l'envoyait au couvent, pour arrêter sans doute son penchant au plaisir, qui s'était déjà déclaré et qui a causé, dans la suite, tous ses malheurs et les miens (p. 20).

Nous sommes devant un fragment de récit, plus précisément devant le récit d'un dialogue. Comme partout ailleurs dans le récit de des Grieux, le locuteur est clairement reconnaissable et la source de son savoir, évidente. Mais la dernière phrase, tout à coup, fait problème. "C'était malgré elle qu'on l'envoyait au couvent" peut être en style direct (le narrateur relate ce qu'il sait des sentiments de sa partenaire), mais se lit tout aussi bien en style indirect libre: le locuteur dans le texte serait des Grieux, mais l'énoncé appartiendrait à Manon. La fin de la phrase appartient évidemment au narrateur: "son penchant au plaisir, qui a causé, dans la suite, tous ses malheurs et les miens." Reste l'entre-deux: "on l'envoyait au couvent, pour arrêter sans doute son penchant au plaisir, qui s'était déjà déclaré." Voilà des choses que le narrateur n'a certainement pas apprises ce jour-là (Manon était bien trop expérimentée), et dont le roman ne nous dit nulle part dans quelles circonstances elles lui auraient été révélées. Vaine question, d'ailleurs: “on l'envoyait au couvent, pour arrêter sans doute son penchant au plaisir"; c'est à une hypothèse du narrateur que nous avons affaire, hypothèse à laquelle se raccroche grammaticalement un autre énoncé ("son penchant au plaisir, qui s'était déjà déclaré"), libre, celui-là, de toute restriction dubitative. Le lecteur reste perplexe, car il reconnaît ici l'une des techniques éprouvées de la diffamation: l'hypothèse désobligeante sur les motifs qu'avaient les Lescaut de mettre leur fille au couvent emprunte sa force à un fait, d'authenticité nébuleuse; le fait, à son tour, gagne en plausibilité d'être intégré à une chaîne causale intelligible: le penchant de Manon au plaisir, qui a causé tous ses malheurs, s'était certainement déclaré fort tôt, et on comprendrait ses parents de l'avoir entourée d'un cordon sanitaire.

Si j'ai parlé de lapsus, c'est que ce genre de perversité dans l'énonciation me semble trancher sur la franchise, l'impudeur même du style de des Grieux. Quant à la portée de la phrase, il est difficile de l'exagérer: "Manon n'a jamais été autre chose qu'une dévergondée," nous dit le narrateur; "c'était un danger public reconnu, comme le confirme la suite de mes malheurs." Des Grieux adopte ici le point de vue de la société sur cette fille "d'une naissance commune," qui prend soin de se faire appeler "Mademoiselle" et qui se trouve "flattée d'avoir fait la conquête d'un amant tel que" lui, d'un gentilhomme (pp. 21-22). Nous sommes assez renseignés sur le lien qu'établit Prévost entre la naissance et les vertus morales pour comprendre qu'à ses yeux aussi, Manon est une ordure. 
L'autre phrase que je voudrais commenter provient de l'épisode américain. On sait que le Nouvel Orléans représente une espèce de paradis de simplicité vertueuse, où l'amour et les bonnes qualités des deux amants peuvent enfin fleurir en toute liberté. D'où le désir qui naît en eux de régulariser leur union. Voici comment des Grieux fait sa proposition:

Nous avons l'âme trop belle, et le coeur trop bien fait, l'un et l'autre, pour vivre volontairement dans l'oubli du devoir. Passe d'y avoir vécu en France, où il nous était également impossible de cesser de nous aimer et de nous satisfaire par une voie légitime; mais en Amérique, où nous ne dépendons que de nous-mêmes, où nous n'avons plus à ménager les lois arbitraires du rang et de la bienséance, où l'on nous croit même mariés, qui empêche que nous ne le soyons bientôt effectivement et que nous n'anoblissions notre amour par des serments que le religion autorise? ( $P$. 190.)

Cette demande en mariage, que Manon n'osait espérer, comble de joie son coeur, où ne règnent plus que l'amour, la religion et l'altruisme.

Nous approchons d'une nouvelle catastrophe, qui donnera à des Grieux l'occasion de gémir sur l'injuste rigueur du Ciel. Il l'annonce en ces termes à son auditeur:

Je suis persuadé qu'il n'y a point d'honnête homme au monde qui n'eût approuvé mes vues dans les circonstances où j'étais, c'est-à-dire asservi fatalement à une passion que je ne pouvais vaincre et combattu par des remords que je ne devais point étouffer (p. 191).

Qui ne voit que ces excuses ruinent de fond en comble toute la construction? Que sert d'avoir fui la France, l'autorité paternelle et les "lois arbitraires du rang et de la bienséance," de ne dépendre que de soi-même; que sert d'avoir trouvé sous des cieux libres une Manon régénérée, qui déteste son passé et qui n'est plus que modération dans la conduite, générosité envers autrui et amour indéfectible pour l'homme de sa vie, s'il faut, pour justifier le mariage avec elle aux yeux d'un honnête homme, plaider les circonstances atténuantes, se dire ligoté par une passion asservissante et torturé par le remords? Ne sommes-nous pas invités à comprendre qu'il y a des choses qu'un honnête homme ne se permet pas, et qu'on n'anoblit pas une Mademoiselle Manon Lescaut?

Lapsus donc, et lapsus de Prévost, indiscutablement, qui consiste à faire avouer au narrateur ce que ni lui, ni nous ne devrions penser, que Prévost lui-même ignorait sans doute, mais qui est le fil conducteur de son récit: jamais, en aucune circonstance il ne pourra s'envisager que Manon soit la femme de des Grieux.

Le Ciel, bien entendu, se déchaîne, mais nous avons compris que ce qu'il pouvait infliger de pire au héros ne serait que la réalisation de son désir. Aussi bien, si nous avions la tentation de prendre la fiction pour la réalité, de voir des faits là où nous lisons en réalité le dévoilement d'un fantasme, il suffirait de considérer un peu attentivement le gouverneur et son neveu Synnelet pour secouer cette illusion: il est trop évident qu'il n'y a point d'autre fatalité à l'oeuvre ici que la chaîne qui lie un homme à son inconscient, puisque cette 
nouvelle paire de personnages reproduit en en redistribuant les traits les figures complices du père, de l'entremetteur, du frère nanti et du rival, déjà vues sous les noms des des Grieux aînés, de Lescaut et des deux G. . . M. . . Plutôt que dans je ne sais quelle obscure métaphysique de la passion et du destin, c'est ici qu'il conviendrait de chercher le tragique du roman (si l'on tient à tout prix à y en trouver), dans l'inaptitude de l'être humain à identifier correctement l'objet de son désir. La réalité est déjà assez dure, pourquoi faut-il que nous ne soyons même pas capables de rêver juste?

Si donc il est exclu que Manon soit la femme de des Grieux, pourrait-elle être une femme pour lui? Il faudrait pour cela qu'il fût un homme, ce qui fait problème, comme nous l'avons vu; et nous avions pu être tentés de croire que le roman narrait la quête d'un phallus artificiel, l'argent, qui aurait levé l'obstacle pour qui eût réussi à le détenir avec assez de constance. Je pense que cette lecture est trop courte et qu'en nous y bornant, nous tomberions dans le même piège que des Grieux, car nous nous ferions accroire que c'est à la présence ou à l'absence de l'argent que tiennent les sentiments de Manon envers lui, théorie fétichiste à laquelle il se cramponne bien que ses aventures, les explications de Manon et l'analyse qu'il fait lui-même de la psychologie de sa maîtresse (par exemple pp. 61-62) lui aient démontré le contraire. Ce qu'il désire, c'est le don d'un corps lié à celui d'un coeur, et c'est cela qui n'entre pas dans la tête de Manon.

Parler de la frigidité de cette héroïne serait étrange, dit Raymond Picard en soulevant la question, dans la préface de l'édition qu'il a donnée du roman avec Frédéric Deloffre; des Grieux ne lui fait-il pas "goûter parfaitement les douceurs de l'amour"? "Mais," enchaîne-t-il,

cette expérience ne semble guère la marquer. L'acte d'amour est pour elle un plaisir parmi d'autres, et qui ne saurait s'inscrire dans un être de façon privilégiée [. . . . Son corps lui reste extérieur [. . . ] (p. cxxii).

Picard rappelle ensuite comment elle croyait satisfaire tout le monde en se donnant au jeune G. . . M. . . et en envoyant une autre fille à des Grieux pour lui faire passer le temps, et ce que fut son étonnement en se rendant compte que son amant n'était pas à l'aise dans un pareil régime d'indifférenciation.

"Son corps lui reste extérieur." La formule est profonde, et Jacques Proust la relève dans sa magnifique étude sur "le Corps de Manon,"4 à qui j'emprunte à mon tour d'importantes observations, que j'essaierai de pousser plus loin à la lumière de ce que j'ai exposé jusqu'ici.

"Que le célèbre roman de Prévost ne contienne aucune référence descriptive à la belle personne qui en est le sujet n'est pas le moindre paradoxe de son oeuvre," dit Proust (p. 107). Cela a été expliqué diversement, et il est probable que le béguin séculaire de milliers de lecteurs pour Manon s'explique en partie par l'espèce de piège que nous tend le texte en dirigeant sur elle un feu nourri de regards admiratifs, de convoitises immédiates et d'épithètes amoureuses, sans permettre au lecteur d'apercevoir autre chose qu'une sorte de brouillard voluptueusement attirant.

Mais le critique ne s'arrête pas là; au lieu de constater simplement le vague, 
il nous montre de quoi il est fait, et révèle la présence de plusieurs complexes de signifiants, dont certains fort inattendus. Au risque de ramener une suite d'analyses très fines à un catalogue décharné, j'énumérerai: le rapport de ce voile que le style de Prévost interpose entre nous et la possible vue de Manon avec le geste de se voiler ou de se cacher qui la caractérise en plusieurs moments-clés du livre; le fait "que son visage soit exsangue chaque fois que le narrateur juge bon de se référer précisément à sa couleur," ce qu'il faut rapprocher de la prédilection pour des mots comme langueur et languissant; un mode d'apparition du personnage dans le décor qui s'apparente à celui des spectres (Proust, pp. 110-111). Ces traits convergent avec le rappel d'une donnée fondamentale, à savoir que toute l'histoire des deux amants se raconte après la mort de Manon, se lit sur l'horizon de cette mort, pour conduire Proust à déclarer que, "si l'auteur ne nous montre jamais le corps de Manon, c'est parce qu'au moment où Renoncour - ou des Grieux - nous en parle, ce ne pourrait être qu'un objet d'effroi et de répulsion [. . . ]. Manon n'est plus qu'un cadavre décomposé," le contraire donc de ce "composé charmant," de "cette figure capable de ramener l'univers à l'idolâtrie" (Prévost, p. 178), qui faisait délirer le héros. Montrant ensuite quelle fonction matricielle la scène de la mort remplit dans le roman, et quelle importance il faut accorder au sable qui semble en résumer tout le décor, Proust conclut: "Dans le contexte, par le contexte, le sable devient un mot poétique, la figure métonymique d'un objet proprement innommable" (pp. 112-113).

"Son corps lui reste extérieur"; peut-être bien parce qu'il n'est pas un corps, mais un agrégat; non pas une unité concrète et vivante, spécifiée comme personne, mais un ramassis de choses, abstraitement réunies par une dénomination ("Manon"), et qui n'attendent qu'un hasard pour se dissocier et ne plus faire qu'un tas de matière innommable.

Désagrégation, décomposition, anonymat, mort, horreur, tabou linguistique: nous avons là des éléments d'une série. J'en trouve le terme suivant au moyen d'un détour par la morale. $\mathrm{Si}$, reprenant le jugement des contemporains et de la postérité, je dis que Manon est une fille, une catin, une perfide, une traînée, une sans coeur, une coureuse, et que je résume en disant qu'elle est une merde, ce n'est pas, qu'on veuille me croire, pour chercher un effet facile. Aucun autre objet de l'expérience humaine ne réunit avec la même netteté les caractéristiques que nous pouvons attribuer à la maîtresse de des Grieux: infiniment précieuse et infiniment abjecte, instrument de jouissance narcissique interdit par les instances parentales, objet perpétuellement reperdu, qui n'est jouissance que dans le risque et le besoin de le perdre, trésor intime et scandale sans nom pour soi-même et pour autrui, matière morte et produit de la vie, image de la mort et de la séparation d'avec soi-même.

Le dernier élément de la série est, évidemment, l'argent, avatar sans odeur, dit-on, du précédent. Des Grieux ne décrit pas les écus qu'il donne à ses domestiques; pourquoi décrirait-il le corps de Manon, qui appartient à tout le monde, qui va et vient de façon aussi imprévisible que les louis d'or qui remplissent aujourd'hui la bourse du joueur et que les valets auront emportés demain, qui sert à acheter des bijoux et à payer des fiacres, qui peut se remplacer par n'importe quel autre corps de valeur équivalente? Manon est 
publique, anonyme, divisible, mobile, morte et souillée comme l'argent. Courir après l'argent pour s'assurer son coeur, c'est chercher un fétiche pour acheter un fétiche, c'est prendre une valeur d'échange pour une valeur d'usage. ${ }^{5}$

Ceux qui auront cru pouvoir me suivre jusqu'ici sont désormais persuadés qu'il n'y a, dans l'Histoire du chevalier des Grieux et de Manon Lescaut, à un certain niveau de la fantasmation, ni homme, ni femme; mais bien un homme castré et un objet fécal qui lui tient lieu de phallus, fonction que nous pouvons déduire de ce qu'à vingt reprises il déclare que la perte de cet objet est pour lui synonyme de mort, ${ }^{6}$ et qu'il est effectivement détruit lorsque cette perte est consommée. Je voudrais maintenant, très rapidement, suggérer une voie de passage vers un autre niveau, mais avec une extrême prudence, car seule la superposition de ce roman-ci avec les autres productions de Prévost donnerait quelque garantie de certitude. Je me conforte, pour le moment, de penser que ce que j'avance est sur la même longueur d'ondes qu'un grand nombre d'observations dégagées par Jean Sgard.

Il y a trois femmes dans ce livre: Manon, sa remplaçante dans l'épisode du jeune G. . . M. . . (sur qui il n'y a rien à dire) et une troisième, qui ne paraît que sur le mode de l'absence. La mère de des Grieux nous est donnée, dans la scène du Luxembourg, comme l'inaccessible, comme celle qui a appartenu au père, celle dont le père interdit même au fils de parler, tant son approche est sacrilège. Elle fait l'objet d'une allusion prodigieusement indirecte, aux premières pages du récit principal, lorsque le narrateur décrit son éblouissement à la vue de Manon: moi, dit-il, "moi qui n'avais jamais pensé à la différence des sexes [. . .]" (p. 19). Il n'a donc jamais pensé qu'il était d'un sexe différent de celui de sa mère? il ignorait donc qu'elle en eût un? Enfin, rappelons la phrase déjà citée: "étant dans ma vingtième année, j'entrais en droit d'exiger ma part du bien de ma mère."

Peut-être bien que ce que le roman raconte, c'est qu'il l'a eue. Intégralement. Il a eu un corps qui ressemblait à s'y méprendre à une femme, et qui était aussi tendre et aussi fidèle qu'une liasse de billets de la banque de Law; et il a eu une morte. Quand, dans une scène (p. 199sqq.) où je suis d'accord avec Jacques Proust (pp. 121-122) pour voir la seule représentation véritable que ce livre nous offre de l'acte d'amour, quand, dis-je, couché à demi nu sur le sable où Manon se décompose, il y tombe dans un orgasme de douleur, il recueille peut-être précisément son legs le plus cher.

Le moment est venu de préciser ce que j'entendais par mon titre. J'ai voulu montrer à l'oeuvre deux genres de fantasmes reliés à l'argent. Les uns sont de l'espèce qu'on est habitué à rencontrer dans les travaux de psychanalyse et de psychocritique; les autres sont également des produits de l'imaginaire, mais nous savons qu'ils furent consciemment entretenus par des groupes humains considérables, et relèvent de ce qu'on appelle l'idéologie. Telle est par exemple l'idée que les privilèges de certaines familles s'expliquent par une supériorité naturelle, que la richesse leur appartient par harmonie préétablie, etc. 
Il me semble avoir fait comprendre assez clairement comment les deux ordres de fantasmes se prêtent l'un avec l'autre à des jeux de métaphorisation: la basse condition et l'immoralité congénitale de Manon, obstacles infranchissables à l'union publique des amants, disent parfaitement, en la voilant, la répugnance du moi à désirer un objet qui, pour lui plaire, doit être ignoble et perdu; l'impossible identification virile ne se marque pas mieux dans l'histoire d' "amour" que dans le statut du héros. Si je n'indique pas de correspondance en sens inverse, cela tient à ce qu'il y a de sommaire et de rapide dans l'essai qu'on vient de lire, et, fondamentalement, à ce que je décris les deux ordres de fantasmes à des niveaux différents: je n'ai pas dépassé, pour ce qui est de l'idéologie, le niveau du manifeste, des attitudes conscientes et explicites, ce qui n'est dans cet ordre, comme dans le psychanalytique, qu'une première et superficielle approche de l'organisation du discours. Même si Prévost s'identifie à des Grieux, comme on a toutes les raisons de le croire, sa vision du monde n'est pas nécessairement identique à celle qu'il pense qu'il a.

Raymond Joly

Université Laval

\section{Notes}

*Je remercie les membres du Cercle de psychocritique (Québec), en particulier Jacqueline Gourdeau et Ernest Pascal, pour les observations qu'ils m'ont permis d'utiliser en rédigeant ce texte.

1 Antoine-François Prévost, le Philosophe anglais ou Histoire de Monsieur Cleveland, éd. Philip Stewart dans Oeuvres, éd. J. Sgard (Grenoble: Presses universitaires de Grenoble, 1977), Il, p. 75.

${ }^{2}$ Prévost, Histoire du chevalier des Grieux et de Manon Lescaut, éd. Frédéric Deloffre et Raymond Picard, Paris, Garnier, 1965, pp. 16-17. Dans la suite, un chiffre entre parenthèses suffira à renvoyer aux pages de cette édition - c'est-à-dire au texte de Prévost, mais aussi aux précieuses notes des éditeurs.

${ }^{3}$ Jean Sgard, Prévost romancier (Paris: Corti, 1968), p. 236.

"Jacques Proust, "le Corps de Manon," dans Jacques Proust, l'Objet et le texte. Pour une poétique de la prose française du XVIII ${ }^{e}$ siècle (Genève, Droz, 1980), pp. 107-126.

${ }^{5}$ Des Grieux, d'ailleurs, est loin d'être insensible aux charmes supplémentaires que l'opulence permet à Manon de se procurer, ni à la valeur marchande de ce corps (et du sien propre). C'est ce que relève Lionel Gossman dans un article (dont je remercie M. Toby Gelfand de me l'avoir signalé): "Prévost's Manon: Love in the new world," Yale French Studies, 40 (1968), 91-102 (94). Ajoutons aux citations de Gossman ce passage: "L'augmentation de nos richesses redoubla notre affection; Vénus et la fortune n'avaient point d'esclaves plus heureux et plus tendres" (66). Voir également la note suivante.

${ }^{6}$ Cet objet sous ses deux formes: Manon ou l'argent pour entretenir Manon (pp. 53, 108, etc.). 